\title{
Selecting Characteristic Raman Wavelengths to Distinguish Liquid Water, Water Vapor, and Ice Water
}

\author{
Sun-Ho Park and Yong-Gi Kim \\ Department of Physics, Kongju National University, Gongju 314-701, Korea \\ Dukhyeon Kim*, Hai-Du Cheong, Won-Seok Choi, and Ji-In Lee \\ Division of Cultural Studies, Hanbat National University, Daejeon 305-719, Korea
}

(Received June 28, 2010 : revised August 2, 2010 : accepted August 16, 2010)

\begin{abstract}
The Raman shift of water vapor is $3657 \mathrm{~cm}^{-1}$, and this Raman signal can be easily separated from other Raman signals or elastic signals. However, it is difficult to make simultaneous Raman measurements on the three phases of water, namely, ice water, liquid water, and water vapor. This is because we must consider the overlap between their Raman spectra. Therefore, very few groups have attempted to make Raman simultaneous measurements even on two elements (water vapor and liquid water, or water vapor and ice water). We have made an effort to find three characteristic Raman wavelengths that correspond to the three phases of water after measuring full Raman spectra of water on particular days that are rainy, snowy or clear. Finally, we have found that the 401-nm, 404-nm, and 408-nm wavelengths are the most characteristic Raman wavelengths that are representative of the water phases when we are using the 355-nm laser wavelength for making measurements.
\end{abstract}

Keywords: LIDAR, Raman spectroscopy, Remote sensing, Water phase, Atmospheric scattering OCIS codes : (280.3640) Lidar; (170.5660) Raman spectroscopy; (280.0280) Remote sensing; (010.7340) Water; (280.1310) Atmospheric scattering

\section{INTRODUCTION}

The phase change in water is emerging as an important subject in atmospheric meteorology and environmental science. The heat emitted and absorbed during a water phase change is important in atmospheric heat circulation. Atmospheric water vapors are the main absorbers of infrared rays [1]. In addition, the water vapor located within $2 \mathrm{~km}$ of the surface of earth have been identified as having the strongest influence on the rise in the ground surface temperature; moreover, even the water vapors in the stratosphere have been found to impact climate change. Therefore, in order to understand cloud microphysics and to predict the changes in weather and climate, it is necessary to measure meteorological factors prior to the development of clouds, such as the concentration distributions of water vapors, droplet size distributions, aerosol, and the phase changes in atmospheric water. Many groups have attempted to measure such meteorological factors by using the multiple-scattering LIDAR technique [2-4]. However, we cannot find any research results on the phase changes in water. Although many researchers have reported on the Raman scattering characteristics of water in laboratory conditions [5-7], there are only a few reports that have investigated scattering in the atmosphere.

A few methods have been applied to measure the phase change in atmospheric water. Melfi and Cooney [8-10] have detected the Raman scattering from water vapor and liquid water by using a Ruby laser.

However, because the Raman spectra of liquid water and ice water cannot be completely resolved, they cannot be used to correctly measure the liquid water content [11]. Veselovskii et al. [12] have used a polarization Raman LIDAR to observe liquid water and water vapor, but they cannot distinguish the phase changes in clouds because they have used wideband interference filters and a wavelength $(401.5 \mathrm{~nm})$ unsuitable for differentiating the two phases because they had no interest in ice water and liquid

\footnotetext{
*Corresponding author: dhkim7575@paran.com

Color versions of one or more of the figures in this paper are available online.
} 
water at that time. Moreover, their study was not aimed at distinguishing ice, liquid, and water vapor. Rizi et al. have also utilized two interference filters to measure liquid water and water vapor [13].

However, the Raman spectra of liquid water and ice water have a broad frequency range of $2800 \mathrm{~cm}^{-1} \sim 3900 \mathrm{~cm}^{-1}$, and these spectra (the Raman spectra of ice and liquid water) overlap in certain areas. Hence, the Raman signals from the liquid water channel contain a large contribution from ice water scattering. In addition, the molecular structure of water under atmospheric conditions is very complex; for example, the water molecules are attached to an aerosol or hydrogen-bonded with other water molecules depending on the temperature and other factors; hence, the shape of the Raman spectrum also very complex. Many researchers have attempted to investigate the microphysics of clouds; they faced limitations in measuring the phase changes in water. For this reason, we must measure full Raman spectra of water in atmospheric conditions, and not in laboratory conditions $[14,15]$, so as to decide the optimum Raman wavelength used in a water Raman LIDAR [16]. In this study, we have designed a Raman LIDAR system based on a monochromator so as to obtain full Raman spectra of atmospheric ice water, liquid water, and water vapor. The final objective of this research is to identify the three optimal wavelengths that are important in designing a filter-based water Raman LIDAR.

\section{EXPERIMENTAL SET UP AND RESULTS}

The Raman spectra of liquid and ice water depend on the number and structure of hydrogen-bonded water molecules $[14,15]$. There has so far been no report about the accurate optimal wavelengths that corresponded to the Raman signals of ice water, liquid water, and water vapor in natural clouds. In order to decide the optimum wavelengths that can distinguish these phases, we have designed a new

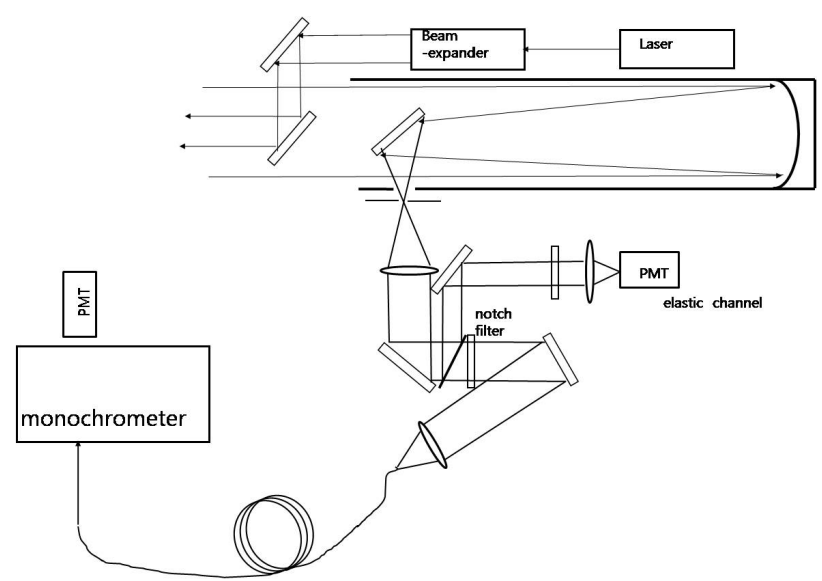

FIG. 1. Experimental setup for measuring Raman spectrum of water.
Raman LIDAR based on a single spectrometer.

Figure 1 shows a schematic diagram of the experimental setup. We used a tripled Nd:YAG laser with an energy $220 \mathrm{~mJ}$ and a repetition rate of $10 \mathrm{~Hz}$. The backscattered signals were collected by using a $320-\mathrm{mm}$ parabolic telescope with a focal length of $1500 \mathrm{~mm}$. In the setup, first the scattered light is collimated by the lens after passing through a pinhole; then the collimated light is passed through a notch filter (Razor Edge Longpass 355) that transmits all the Raman wavelengths but reflects other wavelengths less than $355 \mathrm{~nm}$. The blocking ratio between the transmitted and reflected wavelengths at this notch filter exceeds $10^{-3}$. A signal that has passed through the notch filter is focused on a fiber connected to a monochromator. We have used Acton Research's SpectraPro-500 models, which have a 1200 -grooves $/ \mathrm{mm}$ diffraction plate. The Raman spectrum is recorded in the wavelength range from $398 \mathrm{~nm}$ to $412 \mathrm{~nm}$ with a $1-\mathrm{nm}$ resolution. We have used Hamamatsu R9880U-210, which is specially designed for using in the UV region; its quantum efficiency is $42 \%$ near $400 \mathrm{~nm}$. The Raman signals are measured by using a photon counter. Because a measurement time of $4 \mathrm{~min}$ is required for each wavelength, we need a measuring time of $1 \mathrm{~h}$ to obtain a full Raman spectrum from $398 \mathrm{~nm}$ to $412 \mathrm{~nm}$. In order to measure weather changes during this $1 \mathrm{~h}$, we have monitored the weather condition by measuring elastic signals simultaneously with these Raman signals.

Figure 2 shows Raman spectra of ice, liquid water and water vapor measured under laboratory conditions [17]. However, we cannot confirm whether the shape of this spectrum is the same as that of a full Raman spectrum measured in atmospheric conditions. To the best of our knowledge, these spectra have not been measured in atmospheric conditions, even though D. Kim et al. have measured them in an unknown vertical direction [18].

Therefore, we must decide three characteristic Raman wavelengths after measuring and analyzing full Raman spectra in known atmospheric conditions. All figures (from

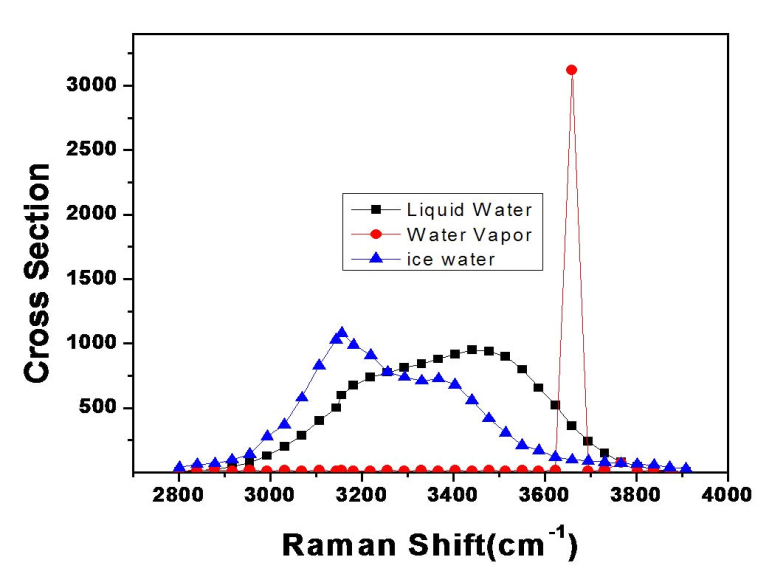

FIG. 2. Shapes of Raman spectra of liquid water, water vapor, and ice $[16,17]$. 


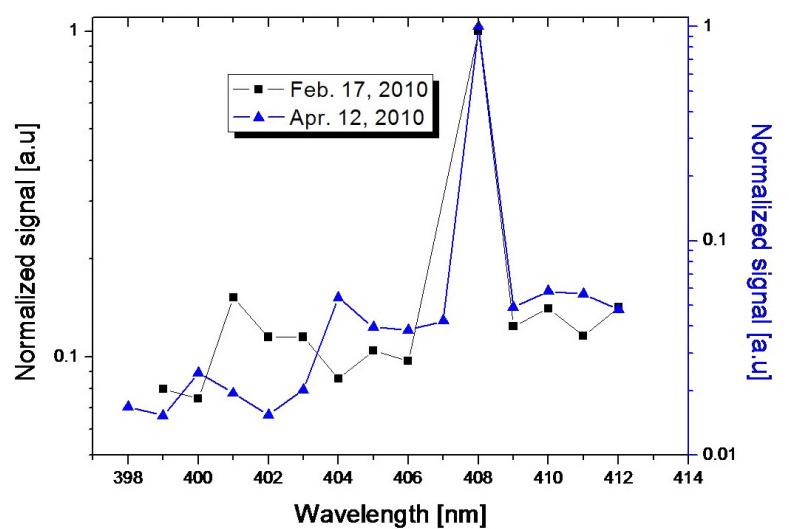

(a)

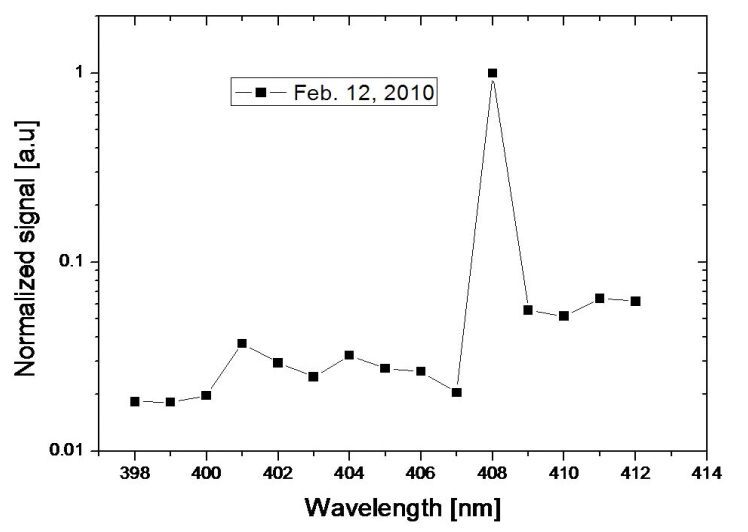

(b)

FIG. 3. Full Raman spectra measured in the horizontal direction (at Hanbat National University in Daejeon, Korea). (a) Feb. 17, 2010 (Snowing), Apr. 12, 2010 (Raining), (b) Feb. 12, 2010 (Mixed rain and snow sleet).

Fig. (3) to Fig. (7)) are normalized by using a water vapor Raman signal to see the changes of the Raman spectrum that depend on the phase of water.

Figure 3(a) shows the full Raman spectra measured in the horizontal direction (at Hanbat National University in Daejeon, Korea), on a rainy day and a snowy day. As this figure shows, we have a peak near $401 \mathrm{~nm}$ on a snowy day (Feb. 12, 2010); on the other hand, we have different peaks near $404 \mathrm{~nm}$ on a rainy day (Apr. 12, 2010). From these results, we have temporarily decided on $401 \mathrm{~nm}$ and $404 \mathrm{~nm}$ as the characteristic wavelengths of ice and liquid water, respectively. Figure 3(b) shows a special Raman spectrum that is measured on a particular day on which rainfall and snowfall occurred simultaneously. As expected, Fig. 3(b) has two peaks, one at $401 \mathrm{~nm}$ and the other at $404 \mathrm{~nm}$. We can consider think that $401 \mathrm{~nm}$ and $404 \mathrm{~nm}$ to be the representative wavelengths of ice and liquid water Raman signals, respectively, when we use a 355-nm laser for making measurements.

We have measured full Raman spectra on a clear day also, but under different temperature and humidity conditions. Figure 4 shows temperature- and humidity-dependent

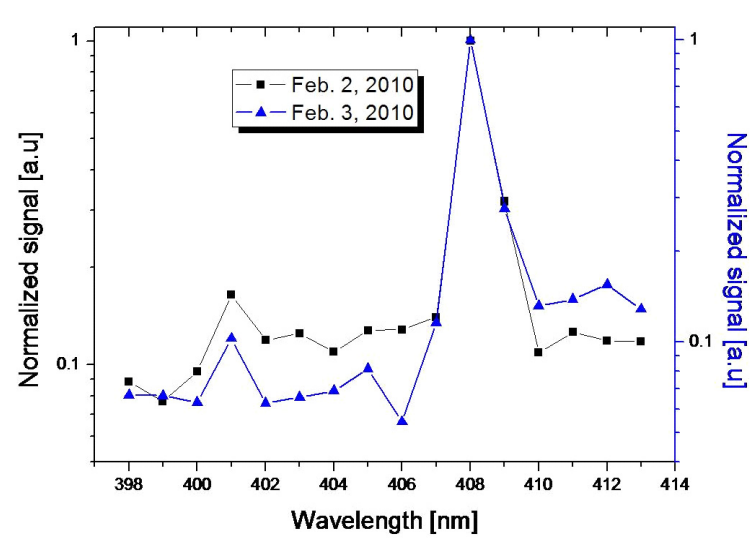

(a)

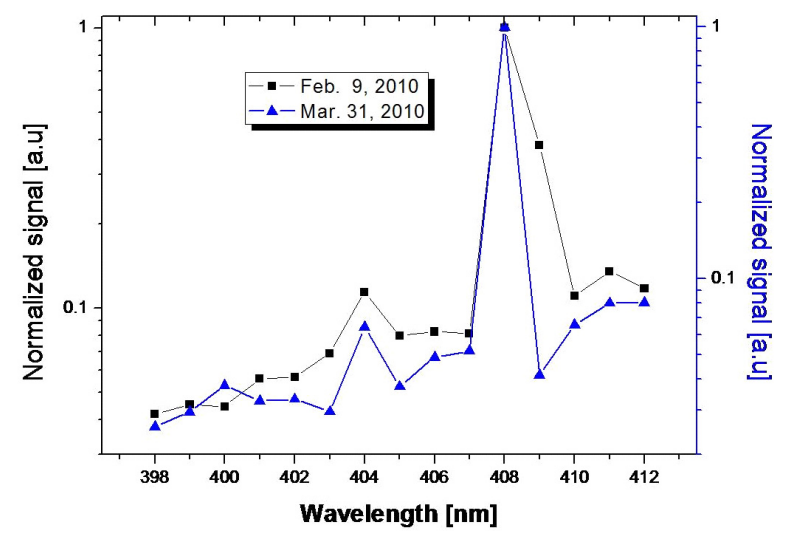

(b)

FIG. 4. Temperature-dependent Raman spectra. (a) measured on Feb. 2 and 3, $2010\left(-6^{\circ} \mathrm{C}(40 \%),-7^{\circ} \mathrm{C}(49 \%)\right)$, (b) measured on Feb. 9 and Mar. 31, $2010\left(5^{\circ} \mathrm{C}(97 \%), 9^{\circ} \mathrm{C}(97 \%)\right)$.

Raman spectra. Figure 4(a) shows the spectra measured on Feb. 2 and Feb. 3, 2010; Fig. 4(b), shows the spectra measured on Feb. 9 and Mar. 31, 2010. The temperature and humidity were $-6^{\circ} \mathrm{C}(40 \%),-7^{\circ} \mathrm{C}(49 \%), 5^{\circ} \mathrm{C}(97 \%)$, and $9^{\circ} \mathrm{C}(97 \%)$ on Feb. 2, Feb. 3, Feb. 9, and Mar. 31, respectively.

All these figures indicate that the Raman wavelength of water vapor is constant (408 nm), and we consistently have a peak at $401 \mathrm{~nm}$ on a low-temperature low-humidity day, and at $404 \mathrm{~nm}$ on a high-temperature high-humidity day.

Intuitively, we can consider that water will be in the ice state on a low-temperature low-humidity day (Feb. 2 and Feb. 3, 2010), and it will be in the liquid state on a hightemperature high-humidity day (Feb. 9, Mar. 31, 2010), and these spectra also represent these facts well. Even though we have not shown other data in this paper, we have obtained similar results in many cases. D. Whiteman [19] has also indicated that $404 \mathrm{~nm}$ is the isosbestic point. Hence, $404 \mathrm{~nm}$ is the best Raman wavelength for measuring the liquid water content (LWC).

Figure 5 shows the full Raman spectrum measured on a foggy day (Mar. 4, 2010). Because the water contained in fog is in the liquid state, we can expect that this spectrum 
should be similar to that recorded on a rainy day, and in accordance with our expectation, the spectrum shapes observed in Fig. 5 and Fig. 3 (a) (Apr. 12, 2010) are almost the same.

Figure 6(a) shows the full Raman spectra measured under the conditions of heavy rain and light rain. Fig. 6(b)

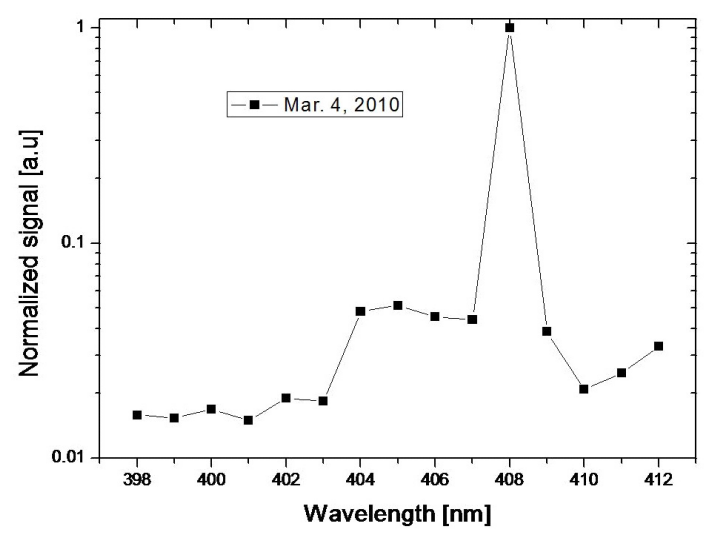

FIG. 5. Full Raman spectrum measured on foggy day (Mar. 4, 2010).

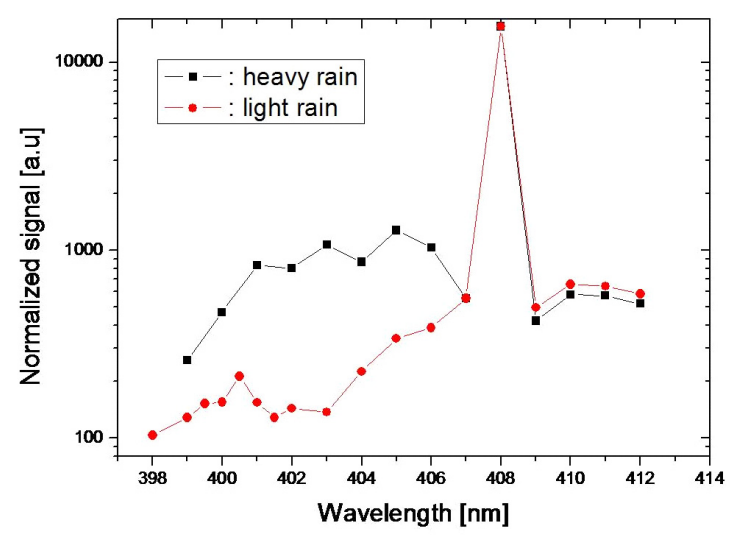

(a)

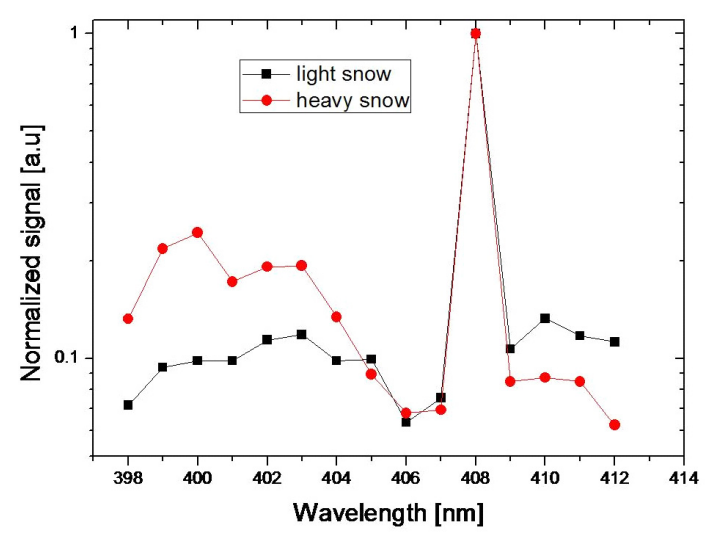

(b)

FIG. 6. (a) Full Raman spectrum measured under conditions of heavy and light rain. (b) measured under the conditions of heavy and light snow. is measured under the conditions of heavy snow and light snow. As these figures show, the number of photons depends on the weather conditions. That is, we can detect more photons under heavy rain than under light rain in the 404-nm. On a snowy day, the same results are obtained for the 401-nm Raman wavelength channel.

To select characteristic Raman wavelengths, we considered following criteria. 1) Raman cross section should be large. 2) To measure the water phase change, phase change criterion $\mathrm{p}$ should change as much as possible when liquid water changes to ice water or vice versa.

$$
p=\frac{S_{\lambda_{1}}^{\text {Raman }}-S_{\lambda_{2}}^{\text {Raman }}}{\left|S_{\lambda_{1}}^{\text {Raman }}+S_{\lambda_{2}}^{\text {Raman }}\right|}
$$

Here $S_{i}^{\text {Raman }}$ is the Raman signal at wavelength I, $\lambda_{1}$ is the characteristic wavelength of ice, and $\lambda_{2}$ is the characteristic wavelength of liquid. 3) Wavelength difference between $\lambda_{1}$ and $\lambda_{2}$ should be as large as possible to easily construct the Raman LIDAR system. 4) Two Raman wavelengths $\lambda_{1}$ and $\lambda_{2}$ should be separated as far as possible from the rotational Raman wavelength of water vapor. From these considerations, we can say that $401 \mathrm{~nm}$ and $404 \mathrm{~nm}$ are the characteristic wavelengths and that by measuring these wavelengths, we can simultaneously measure LWC and ice water content (IWC).

Figure 7 shows a schematic diagram of our filter-based 5-channel Raman LIDAR system. The system has nitrogen Raman (386 nm), ice water Raman $(401 \mathrm{~nm})$, liquid water $(404 \mathrm{~nm})$, water vapor $(408 \mathrm{~nm})$, and elastic $(355 \mathrm{~nm})$ channels. In order to normalize time-dependent atmospheric conditions and laser energy changes, we have divided each water Raman signal with the nitrogen Raman signal.

Figure 8 shows traditional normalized Raman LIDAR signals measured on warm and cold days in the vertical direction. These signals are normalized by using a nitrogen Raman signal to see relative concentrations of water

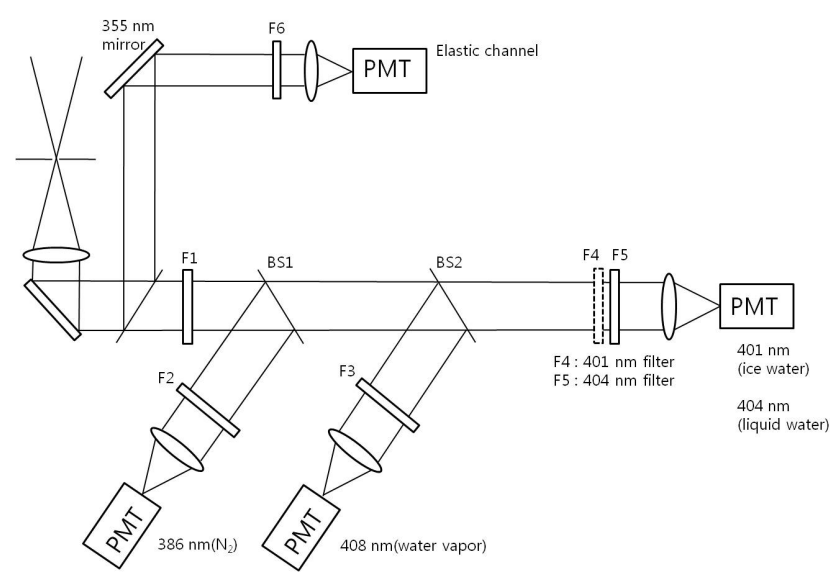

FIG. 7. Schematic diagram of our filter-based 5-channel Raman LIDAR system. 
vapor, liquid water, and ice water. These signals have been obtained on Oct. 20, 2009, Nov. 13 and 16, 2009. The respective temperatures on these days were $10^{\circ} \mathrm{C}$, $10.3^{\circ} \mathrm{C}$ (Fig. 8(a)) and $0^{\circ} \mathrm{C}$ (Fig. 8(b)) respectively. On a warm day (Fig. 8(a)), we can detect clouds at distances of $1.5 \mathrm{~km}$ and $2.3 \mathrm{~km}$, and the clouds at these distances scatter relatively stronger Raman signals of water vapor and liquid water than of ice water. On the other hand, on a cold day (Fig. 8(b)), we can consider that a cloud is composed of ice water. Experimental results also show that the ice Raman signal $(401 \mathrm{~nm})$ is stronger than the water vapor Raman signal. When we consider the surface temperature of our region, humidity, and altitude of the cloud, these results indicate that our filter-based Raman LIDAR system can give information on cloud phase, LWC, and IWC.

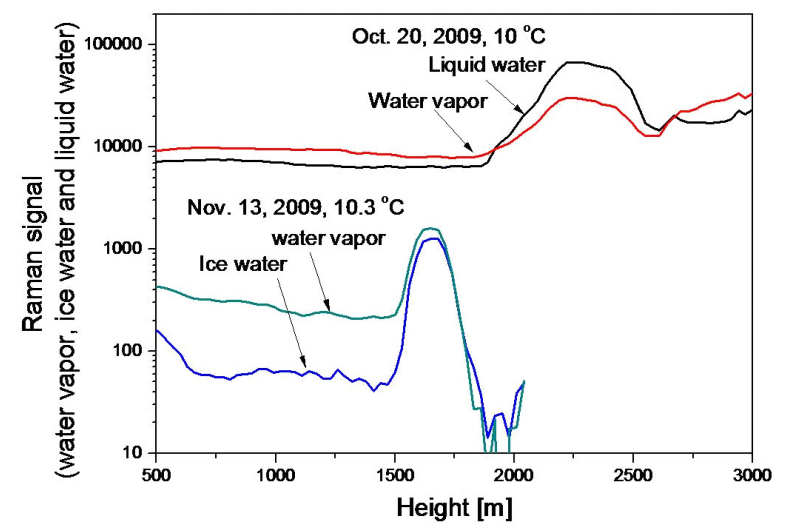

(a)

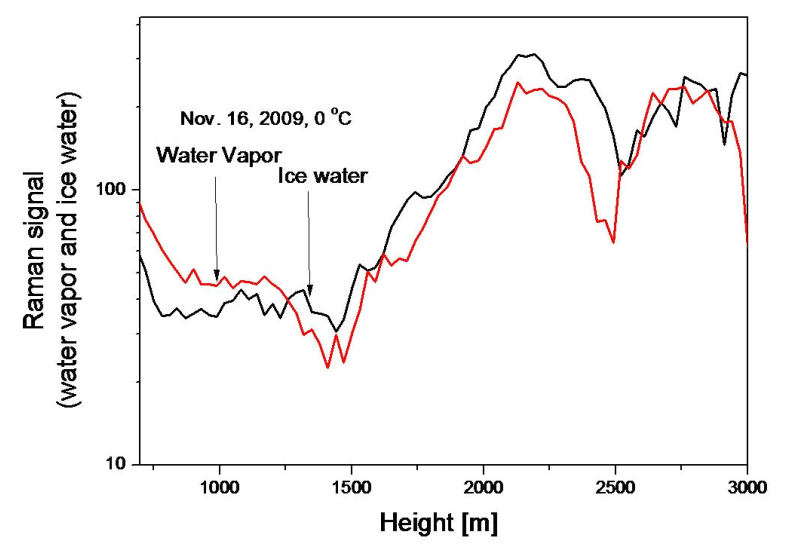

(b)

FIG. 8. Traditional normalized Raman LIDAR signal measured on a cloudy day in vertical direction. (a) On warm days, the ratios of water vapor, ice water and liquid water Raman signals to nitrogen Raman signal ( $P_{\text {Vapor }} / P_{\text {Nitrogen, }}$ $P_{\text {Ice }} / P_{\text {Nitrogen }}$ and $\left.P_{\text {Liquid }} / P_{\text {Nitrogen }}\right)$ measured on Oct. 20, 2009 and Nov. $13,2009\left(10^{\circ} \mathrm{C}, 10.3^{\circ} \mathrm{C}\right)$. (b) On a cold day, ratio of ice water and water vapor Raman signals to nitrogen Raman signal $\left(P_{\text {Vapor }} / P_{\text {Nitrogen }}, P_{\text {Ice }} / P_{\text {Nitrogen }}\right)$ measured on Nov. 16, $2009\left(0^{\circ} \mathrm{C}\right)$.

\section{DISCUSSION AND CONCLUSIONS}

In this study, we have developed a Raman LIDAR system that can distinguish the Raman spectra of ice water, liquid water, and water vapor. This LIDAR system is composed of a notch filter and a monochromator. By using the system we have found and confirmed the optimum Raman wavelengths that are representative of water phases.

We have measured Raman LIDAR spectra on a rainy day and a snowy day and compared them. From these two representative spectra, we can obtain characteristic Raman wavelengths (401 nm, $404 \mathrm{~nm}$, and $408 \mathrm{~nm}$ ) that can provide quantitative information for determining the content of ice water, liquid water, and water vapor in clouds and atmospheric conditions.

In order to prove these characteristic wavelengths, we have obtained full Raman LIDAR signals on a clear warm day and a cold day (Feb. 2 and Feb. 9, 2010, respectively). These full Raman signals also show the same characteristic wavelengths (401 nm, $404 \mathrm{~nm}$, and $408 \mathrm{~nm}$ ) as those recorded on rainy and snowy days. The full Raman spectrum measured on a foggy day differs completely from that recorded on a snowy day, but more or less looks like the one measured on a rainy day.

After finding these characteristic Raman wavelengths, we have analyzed the filter-based Raman LIDAR signals; these signals were measured from a cloud at three characteristic wavelengths (401 nm, $404 \mathrm{~nm}$, and $408 \mathrm{~nm}$ ).

As a standard example, we have compared the LIDAR signals of an ice cloud and a liquid cloud, which were obtained on Nov. 16 and Oct. 20, 2009. These signals also show that our characteristic wavelengths are suitable for distinguishing the Raman signals from liquid water and ice water.

As mentioned above, we have first found three characteristic LIDAR Raman wavelengths $(401 \mathrm{~nm}, 404 \mathrm{~nm}$, and $408 \mathrm{~nm}$ ). In the near future, we will apply the filter-based LIDAR system to characterize the hygroscopic properties of atmospheric aerosol and to investigate the true state of a cloud.

\section{ACKNOWLEDGMENT}

This work was funded by the Korea Meteorological Administration Research and Development Program under grant No. CATER 2008-3109.

\section{REFERENCES}

1. V. Ramaswamy and S. M. Freidenriech, "Solar radiative line-by-line determination of water vapor absorption and water cloud extinction in inhomogeneous atmospheres," J. 
Geophys. Res. 96, 9133-9157 (1991).

2. J. A. Weinman, "Monte Carlo analysis of multiple scattering on light pulses reflected by turbid atmospheres," J. Atmos. Sci. 33, 1763-1771 (1976).

3. S. T. Shipley and D. H. Tracy, "High spectral resolution LIDAR to measure optical scattering properties of atmospheric aerosols 1: theory and instrumentation," Appl. Opt. 22, 3716-3724 (1983)

4. J. T. Sroga, E. W. Eloranta, S. T. Shipley, F. L. Roesler, and P. J. Tryon, "High spectral resolution LIDAR to measure optical scattering properties of atmospheric aerosols. 2: calibration and data analysis," Appl. Opt. 22, 3725-3732 (1983).

5. J. R. Scherer, M. K. Go, and S. Kint, "The vibrational spectroscopy of water," J. Phys. Chem. 78, 1304-1313 (1974).

6. G. E. Walrafen, "Raman spectral studies of the effects of temperature on water structure," J. Chem. Phys. 47, 114126 (1967).

7. G. Schweiger, "Raman scattering on microparticles: size dependence," J. Opt. Soc. Am. B 8, 1770-1778 (1991).

8. S. H. Melfi, "Observation of atmospheric fronts using Raman LIDAR moisture measurements," J. Appl. Meteor. 28, 789-806 (1989).

9. S. H. Melfi, J. D. Lawrence Jr., and M. P. McCormick, "Observation of Raman scattering by water vapor in the atmosphere," Appl. Phys. Lett. 15, 295-297 (1963).

10. J. A. Cooney, "Measurement of atmospheric temperature profiles by Raman backscatter," J. Appl. Meteor. 11, 108112 (1972).

11. S. H. Melfi, K. D. Evans, J. Li, D. Whiteman, R. Ferrare, and G. Schwemmer, "Observation of Raman scattering by cloud droplets in the atmosphere," Appl. Opt. 36, 3551-3559 (1997).
12. I. A. Veselovskii, H. K. Cha, D. H. Kim, S. C. Choi, and J. M. Lee, "Study of atmospheric water in gaseous and liquid state by using combined elastic-Raman depolarization LIDAR," Appl. Phys. B 73, 739-744 (2001).

13. V. Rizi, M. Iarlori, G. Rocci, and G. Visconti, "Raman LIDAR observations of cloud liquid water," Appl. Opt. 43, 6440-6453 (2004).

14. A. Ansmann, M. Riebesell, U. Wandinger, C. Weitlamp, E. Voss, and W. Michaelis, "Combined Raman elastic-backscatter LIDAR for vertical profiling of moisture, aerosol extinction, backscatter, and LIDAR ratio," Appl. Phys. B 55, 18-28 (1992).

15. M. F. Vernon, D. J. Krajnovich, H. S. Kwok, J. M. Lisy, Y. R. Shen, and Y. T. Lee, "Infrared vibrational predissociation spectroscopy of water clusters by the crossed laser molecular beam technique," J. Chem. Phys. 77, 47-57 (1982).

16. G. D. A'arrigo, G. Maisano, F. Mallamace, P. Migliadrdo, and F. Wanderingh, "Raman scattering and structure of normal and supercooled water," J. Chem. Phys. 75, $4264-4270$ (1981).

17. N. P. Andreeva, A. F. Bunkin, and S. M. Persin, "Deformation of the Raman scattering spectrum of ice under local laser heating near 0 degree," Optics and Spectroscopy 93, 252-256 (2002).

18. D. Kim, I. Song, H. D. Cheong, Y. Kim, S. Baik, and J. Lee, "Spectrum characteristics of multichannel water Raman LIDAR signals and principal component analysis," Opt. Rev. 17, 84-89 (2010).

19. D. H. Whiteman, G. E. Walrafen, W. H. Yang, and S. H. Melfi, "Measurement of an isosbestic point in the Raman spectrum of liquid water by use of a backscattering geometry," Appl. Opt. 38, 2614-2615 (1999). 